\title{
Controllability of Nonlinear Neutral Stochastic Differential Inclusions with Infinite Delay
}

\author{
Yong $\mathrm{Li}^{1}$ and Qiang $\mathrm{Zou}^{2}$ \\ ${ }^{1}$ College of Mathematics Science, Chongqing Normal University, Chongqing 400047, China \\ ${ }^{2}$ School of Computer and Information Science, Southwest University, Chongqing 400715, China \\ Correspondence should be addressed to Yong Li; yongli260@163.com
}

Received 17 November 2012; Revised 20 February 2013; Accepted 15 March 2013

Academic Editor: Yong-Kui Chang

Copyright (c) $2013 \mathrm{Y}$. Li and Q. Zou. This is an open access article distributed under the Creative Commons Attribution License, which permits unrestricted use, distribution, and reproduction in any medium, provided the original work is properly cited.

\begin{abstract}
The paper is concerned with the controllability of nonlinear neutral stochastic differential inclusions with infinite delay in a Hilbert
\end{abstract} space. Sufficient conditions for the controllability are obtained by using a fixed-point theorem for condensing maps due to O'Regan.

\section{Introduction}

Let $K$ be a separable Hilbert space, and let $\left(\Omega, \mathfrak{F}, \mathfrak{F}_{t}, P\right)$ be a complete probability space furnished with a complete family of right continuous increasing $\sigma$ algebras $\left\{\mathfrak{\mho}_{t}\right\}$ satisfying $\mathfrak{F}_{t} \subset$ $\mathfrak{F}$ for $t \geq 0$. Suppose $w(t)$ is a given $K$-valued, $\mathfrak{F}_{t}$ adapted Brownian motion with a finite trace nuclear covariance operator $Q \geq 0$. We are interested in the controllability of nonlinear stochastic differential inclusions with infinite delay

$$
\begin{aligned}
& d\left[x(t)-f\left(t, x_{t}\right)\right] \\
& \quad \in A x(t) d t+(B u)(t) d t \\
& +G\left(t, x_{t}\right) d w(t), \quad t \in J=[0, b], t>0, \\
& \quad x_{0}=\phi \in \mathfrak{B}_{h}, \quad t \in J_{0}=(-\infty, 0],
\end{aligned}
$$

where $\phi$ is $\mathfrak{F}_{0}$ measurable and $A$ is the infinitesimal generator of an analytic semigroup of bounded linear operator $T(t)$, $t \geq 0$, the state $x(\cdot)$ takes values in Hilbert space $H$ with the norm $|\cdot|, G: J \times \mathfrak{B}_{h} \rightarrow 2^{L(K, H)}$ is a bounded closed, convexvalued multivalued map, $f: J \times \mathfrak{B}_{h} \rightarrow H$ is continuous, and the histories $x_{t}:(-\infty, 0] \rightarrow H, x_{t}(\theta)=x(t+\theta)$, and $\theta \leq 0$ belong to the space $\mathfrak{B}_{h}$. For $\sigma_{1}, \sigma_{2} \in L(K, H)$, define $\left\langle\sigma_{1}, \sigma_{2}\right\rangle=\operatorname{tr}\left(\sigma_{1} Q \sigma_{2}^{*}\right)$, where $\sigma_{2}^{*}$ is the adjoint of the operator $\sigma_{2}, Q \in L_{n}^{+}(K) . L(K, H)$ furnished with the scalar product $\langle\cdot, \cdot\rangle$ is a pre-Hilbert space. The completion of $L(K, H)$ with respect to the topology induced by the norm $\|\cdot\|_{2}$, where $\|\sigma\|_{2}^{2}=\langle\sigma, \sigma\rangle_{2}$, is a Hilbert space.

At first, we present the abstract phase space $\mathfrak{B}_{h}$. Assume that $h:(-\infty, 0] \rightarrow(0,+\infty)$ is continuous function with $l=\int_{-\infty}^{0} h(s) d s<+\infty$. Define

$$
\mathfrak{B}_{h}=\{\varphi:(-\infty, 0] \longrightarrow H:
$$

for any $a>0,\left(E|\varphi(\theta)|^{2}\right)^{1 / 2}$ is a bounded and measurable function on $[-a, 0]$ and

$$
\left.\int_{-\infty}^{0} h(s) \sup _{s \leq \theta \leq 0}\left(E|\varphi(\theta)|^{2}\right)^{1 / 2} d s<+\infty\right\} \text {. }
$$

If $\mathfrak{B}_{h}$ is endowed with the norm

$$
\|\phi\|_{\mathfrak{B}_{h}}=\int_{-\infty}^{0} h(s) \sup _{s \leq \theta \leq 0}\left(E|\phi(\theta)|^{2}\right)^{1 / 2} d s, \quad \forall \phi \in \mathfrak{B}_{h},
$$

then $\left(\mathfrak{B}_{h},\|\cdot\|_{\mathfrak{B}_{h}}\right)$ is a Banach space.

Controllability is one of the fundamental concepts in mathematical control theory and plays an important role in control systems. The problem of controllability is to show the existence of a control function, which steers the solution of the system from its initial state to a final state, where the initial and final states may vary over the entire space. Controllability 
generally means that it is possible to steer a dynamical control system from an arbitrary initial state to an arbitrary final state using the set of admissible controls. A standard approach is to transform the controllability problem into a fixed-point problem for an appropriate operator in a functional space. The problem of controllability for functional differential systems has been extensively studied in many papers [1-10]. For example, Sakthivel et al. [5] considered a class of fractional neutral control systems governed by abstract nonlinear fractional neutral differential equations and established a new set of sufficient conditions for the controllability of nonlinear fractional systems by using a fixed-point analysis approach. Using fixed-point technique, fractional calculations, stochastic analysis technique, and methods adopted directly from deterministic control problems, Sakthivel et al. [7] gave a new set of sufficient conditions for approximate controllability of fractional stochastic differential equations. In [8], Sakthivel and Ren investigated the complete controllability property of a nonlinear stochastic control system with jumps in a separable Hilbert space. In [9], Sakthivel et al. studied the approximate controllability for nonlinear dispersion system under the assumption that the corresponding linear control system is approximately controllable.

Benchohra et al. [11] discussed the controllability for oneorder, second-order functional differential and integrodifferential inclusions in Banach space with finite delay. Park et al. [12] discussed the controllability for second-order neutral functional differential inclusions in Banach space with the help of some fixed-point theorems. In $[13,14]$, Liu investigated the controllability of neutral functional differential and integrodifferential inclusions with infinite delay.

History about the research of stochastic differential equations is over fifty years; many important results on the stochastic differential equations have been reported in the literature; see $[15,16]$. Recently, stochastic partial differential equations (SPDE) arise in the mathematical modeling of various fields in physics and engineering science cited by Sobczyk [16]. About neutral stochastic differential equations with delay, Mahmudov [17] discussed the existence and uniqueness of neutral stochastic differential equations by using Picard technique. For the controllability of stochastic functional differential inclusions, Balasubramaniam and Ntouyas [18] discussed the controllability of semilinear stochastic delay evolution equations in Hilbert spaces. Balasubramaniam and Dauer [19] obtained the controllability result of stochastic differential inclusions with infinite delay in abstract space. Since many systems arising from realistic models heavily depend on histories (i.e., there is the effect of infinite delay on state equations) [20], there is a real need to discuss partial functional differential systems with infinite delay. So in the present paper, we will concentrate on the case with infinite delay and establish sufficient conditions for the controllability of systems (1) by relying on a fixed-point theorem for condensing maps due to O'Regan [21].

The structure of this paper is as follows. In Section 2 we briefly present some basic notations and preliminaries. The controllability result of system (1) is investigated by means of fixed-point theorem and operator theory in Section 3. Conclusion is given in Section 4.

\section{Preliminaries}

Let $(E,\|\cdot\|)$ be a Banach space. A multivalued map $\mathfrak{\Im}: E \rightarrow$ $2^{E}$ is convex (closed)-valued, if $\mathfrak{J}(x)$ is convex (closed) for all $x \in E . \mathfrak{I}$ is bounded on bounded set if $\mathfrak{F}(B)=\cup_{x \in B} \mathfrak{J}(x)$ is bounded in $E$ for any bounded set $B$ of $E$; that is,

$$
\sup _{x \in B} \sup \{\|y\| \in \mathfrak{I}(x)\}<\infty .
$$

$\mathfrak{J}$ is called upper semicontinuous (Usc) on $E$ if for each $x_{*} \in E$, the set $\mathfrak{\Im}\left(x_{*}\right)$ is nonempty, closed subset of $E$ and if for each open set $B$ of $E$ containing $\mathfrak{J}\left(x_{*}\right)$, there exists an open neighborhood $V$ of $x_{*}$ such that $\mathfrak{\Im}(V) \subseteq B$.

$\mathfrak{J}$ is said to be completely continuous if $\mathfrak{J}(B)$ is relatively compact, for every bounded subset $B \subseteq E$.

If the multivalued map $\mathfrak{\Im}$ is completely continuous with nonempty compact values, then $\mathfrak{I}$ is Usc if and only if $\mathfrak{J}$ has a closed graph (i.e., $x_{n}=x_{*}, y_{n}=y_{*}, y_{n} \in \mathfrak{J} x_{n}$ imply $y_{*} \in$ $\left.\mathfrak{\Im} x_{*}\right)$.

Let $\mathrm{BCC}(E)$ denote the sets of all the sets of all nonempty, bounded, closed, and convex subsets of $E$. For more details on multivalued maps see the books of Deimling [22] and $\mathrm{Hu}$ and Papageorgiou [23].

An upper semicontinuous map $H: E \rightarrow E$ is said to be condensing if for any subset $B \subseteq E$ with $\alpha(B) \neq 0$, we have $\alpha(H(B))<\alpha(B)$, where $\alpha$ denotes the Kuratowski measure of noncompactness. It is easy to see that $s$ completely continuous multivalued map is a condensing map.

If $T$ is a uniformly bounded and analytic semigroup with infinitesimal generator $A$ such that $0 \in \rho(A)$ then it is possible to define the fractional power $(-A)^{\alpha}$, for $0<\alpha \leq 1$, as a closed linear operator on its domain $D\left((-A)^{\alpha}\right)$. Furthermore, the subspace $D\left((-A)^{\alpha}\right)$ is dense in $X$ and the expression

$$
\|x\|_{\alpha}:=\left\|(-A)^{\alpha} x\right\|, \quad x \in D\left((-A)^{\alpha}\right)
$$

defines a norm on $D\left((-A)^{\alpha}\right)$. Hereafter we represent by $X_{\alpha}$ the space $D\left((-A)^{\alpha}\right)$ endowed with the norm $\|\cdot\|_{\alpha}$. Then the following properties are well known [24].

Lemma 1 (see [24]). Suppose that the preceding conditions are satisfied.

(a) Let $0<\alpha \leq 1$. Then $X_{\alpha}$ is a Banach space.

(b) If $0<\gamma<\alpha \leq 1$, then $X_{\alpha} \hookrightarrow X_{\gamma}$ and the imbedding is compact whenever the resolvent operator of $A$ is compact.

(c) For every $a>0$, there exists a positive constant $c_{\alpha}$ such that

$$
\left\|(-A)^{\alpha} T(t)\right\| \leq \frac{c_{\alpha}}{t^{\alpha}}, \quad 0<t \leq a .
$$

The key tool in our approach is following fixed-point theorem [21].

Theorem 2 (see [21]). Let $X$ be a Hilbert space, and let $U$ be an open, convex subset of $X$ and $u_{0} \in U$. If

(a) $F: \bar{U} \rightarrow \mathscr{P}_{c d}(X)$ has closed graph, 
(b) $F: \bar{U} \rightarrow \mathscr{P}_{c d}(X)$ is condensing operator and $F(\bar{U})$ is bounded set of $X$,

then either

(i) F has fixed-point in $\bar{U}$; or

(ii) there exist $u \in \partial U$ and $\lambda \in(0,1)$ such that $u \in \lambda F(u)+$ $(1-\lambda)\left\{u_{0}\right\}$.

\section{Main Result}

In the following, we will apply Theorem 2 to study the existence of solutions for system (1).

Definition 3. A function $x:(-\infty, b] \rightarrow H$ is called a mild solution of system (1) if the following holds: let $\phi$ be $\mathfrak{F}_{0}$ measurable $H$-valued stochastic processes $x_{0}=\phi \in \mathfrak{B}_{h}$ on $(-\infty, 0]$ and the integral equation

$$
\begin{aligned}
x(t)= & T(t)[\phi(0)-f(0, \phi)]+f\left(t, x_{t}\right) \\
& +\int_{0}^{t} A T(t-s) f\left(s, x_{s}\right) d s+\int_{0}^{t} T(t-s)(B u)(s) d s \\
& +\int_{0}^{t} T(t-s) g(s) d w(s), \quad \text { for a.e. } t \in J, \\
x_{0}= & \phi \in \mathfrak{B}_{h}, \quad t \in J_{0}
\end{aligned}
$$

is satisfied, where

$$
g \in S_{G, x}=\left\{g \in L^{2}(J, H): g(t) \in G\left(t, x_{t}\right) \text {, for a.e. } t \in J\right\} \text {. }
$$

Definition 4. System (1) is said to be controllable on the interval $J$ if for every continuous initial random process $\phi \epsilon$ $\mathfrak{B}_{h}, x_{1} \in H$, there exists a control $u \in L^{2}(J, U)$ such that the mild solution $x(t)$ of (1) satisfies $x(b)=x_{1}$.

To investigate the controllability of system (1), we use the following hypotheses.

$\left(H_{1}\right) A$ is the infinitesimal generator of an analytic semigroup of bounded linear operators $T(t)$ in $H, 0 \in$ $\rho(A)$, for $t>0$; there exist constants $M_{1}$ such that $|T(t)| \leq M_{1}$.

$\left(H_{2}\right)$ The linear operator $W: L^{2}(J, U) \rightarrow H$ defined by

$$
W u=\int_{0}^{b} T(b-s) B u(s) d s
$$

has an induced inverse operator $W^{-1}$ which takes values in $L^{2}(J, U) / \operatorname{ker} W$ and there exist positive constants $M_{3}, M_{4}$ such that $|B| \leq M_{3}$ and $\left|W^{-1}\right| \leq$ $M_{4}$.

$\left(H_{3}\right)$ There exist constants $0 \leq c_{1}<1,1 / 2<\beta<1$, and $c_{2} \geq 0$ such that $l^{2} c_{1}\left\|(-A)^{-\beta}\right\|^{2}<1 / 5$ and

$$
E\left|(-A)^{\beta} f(t, u)\right|^{2} \leq c_{1}\|u\|_{\mathfrak{B}_{h}}^{2}+c_{2}, \quad t \in J, u \in \mathfrak{B}_{h},
$$

where $l=\int_{-\infty}^{0} h(s) d s<+\infty$.
$\left(H_{4}\right) G: J \times \mathfrak{B}_{h} \rightarrow \operatorname{BCC}(H) ;(t, \phi) \rightarrow G(t, \phi)$ is measurable with respect to $t$ for each $\phi \in \mathfrak{B}_{h}$, Usc with respect to $\phi$ for each $t \in J$, and for each fixed $\phi \in \mathfrak{B}_{h}$, the set

$S_{G, x}=\left\{g \in L^{2}(J, H): g(t) \in G\left(t, x_{t}\right)\right.$, for a.e. $\left.t \in J\right\}$

is nonempty.

$\left(H_{5}\right) E\|G(t, \phi)\|_{2}^{2}=\sup \left\{E\|v\|_{2}^{2}: \quad v \in G(t, \phi)\right\} \leq$ $p(t) \psi\left(\|\phi\|_{\mathfrak{B}_{h}}\right)^{2}, t \in J, p \in L^{2}\left(J, R^{+}\right), \phi \in \mathfrak{B}_{h}$, and $\psi:$ $[0,+\infty) \rightarrow(0,+\infty)$ is a continuous nondecreasing function. There exists a positive constant $r$ such that

$$
\frac{r}{F^{*}+\bar{M} r+k_{3} \psi\left(3 l^{2} r+3 q^{*}\right) \int_{0}^{b} p(s) d s}>1,
$$

where

$$
\begin{gathered}
F=5 M_{1}^{2}\left\|(-A)^{-\beta}\right\|^{2}\left(c_{1}\|\phi\|_{B_{h}}^{2}+c_{2}\right)+5\left\|(-A)^{-\beta}\right\|^{2} c_{2} \\
+\frac{5 b^{2 \beta} c_{2} c_{1-\beta}^{2}}{2 \beta-1}+25 b^{2} M_{1}^{2} M_{3}^{2} M_{4}^{2} \\
\times\left[\begin{array}{c}
E\left|x_{1}\right|^{2}+2 M_{1}^{2} E|\phi(0)|^{2}+2 M_{1}^{2}\left\|(-A)^{-\beta}\right\|^{2} \\
\left.\times\left(c_{1}\|\phi\|_{B_{h}}^{2}+c_{2}\right)+\left\|(-A)^{-\beta}\right\|^{2} c_{2}+\frac{b^{2 \beta-1} c_{2} c_{1-\beta}^{2}}{2 \beta-1}\right], \\
q^{*}=\|\phi\|_{B_{h}}^{2}+l^{2} M_{1}^{2} E|\phi(0)|^{2}, \\
F^{*}=F+k_{1} q^{*}+\frac{k_{2} b^{2 \beta-1}}{2 \beta-1} q^{*}, \\
k_{3}=5 M_{1}^{2}+25 b^{2}\left(M_{1} M_{2} M_{3} M_{4}\right)^{2} . \\
k_{2}=15 b c_{1} c_{1-\beta}^{2}+75 b^{2} M_{1}^{2} M_{3}^{2} M_{4}^{2}, \\
k_{1}=15\left\|(-A)^{-\beta}\right\|^{2} c_{1}+75 b^{2} M_{1}^{2} M_{3}^{2} M_{4}^{2}\left\|(-A)^{-\beta}\right\|^{2} c_{1}, \\
2 \beta-1
\end{array},\right.
\end{gathered}
$$

$\left(H_{6}\right)$ The operator $F$ with values $(F(x))(t)=f\left(t, x_{t}\right), t \in$ $J, F$ is completely continuous in $C(J, H)$ and for any bounded set $V \subseteq C(J, H)$, the set $\left\{t \rightarrow f\left(t, x_{t}\right): x \in\right.$ $V\}$ is equicontinuous in $C(J, H)$.

$\left(H_{7}\right)$ There exist constants $1 / 2<\beta<1$ and $q>0$ such that $(-A)^{\beta} f$ is continuous and for $t \in J, \phi_{1}, \phi_{2} \in$ $\mathfrak{B}_{h}$ such that $\left|(-A)^{\beta} f\left(t, \phi_{1}\right)-(-A)^{\beta} f\left(t, \phi_{2}\right)\right| \leq$ $q\left\|\phi_{1}-\phi_{2}\right\|_{\mathfrak{B}_{h}}, 0<Q=2\left\|(-A)^{-\beta}\right\|^{2} q^{2}+2 c_{1-\beta}^{2}(1 /(2 \beta-$ 1)) $b^{2 \beta-1} q^{2} l^{2}<1$. 
Lemma 5 (Lasota and Opial [25]). Let I be a compact real interval and let $E$ be a Banach space. Let $G$ be a multivalued map satisfying $\left(H_{4}\right)$ and let $\Gamma$ be a linear continuous mapping from $L^{2}(I, E)$ to $C(I, E)$. Then the operator

$$
\begin{aligned}
& \Gamma \circ S_{G}: C(I, E) \longrightarrow \operatorname{BCC}(C(I, E)), \\
& x \longrightarrow\left(\Gamma \circ S_{G}\right)(x)=\Gamma\left(S_{G, x}\right)
\end{aligned}
$$

is a closed graph operator in $C(I, E) \times C(I, E)$.

Lemma 6 (see [26]). Let $v(\cdot), r(\cdot):[0, T] \rightarrow[0, \infty)$ be continuous functions. If $r(\cdot)$ is nondecreasing and there are constants $\theta>0,0<\tau<1$ such that

$$
v(t) \leq r(t)+\theta \int_{0}^{t} \frac{v(s)}{(t-s)^{1-\tau}} d s, \quad t \in[0, T],
$$

then

$$
v(t) \leq e^{\theta^{n} \Gamma(\tau)^{n} t^{n \tau} / \Gamma(n \tau)} \sum_{j=0}^{n-1}\left(\frac{\theta T^{\tau}}{\tau}\right)^{j} r(t),
$$

for every $t \in[0, T]$ and every $n \in N$ such that $n \tau>1$, and $\Gamma(\cdot)$ is the Gamma function.

Now we consider the space $\mathfrak{B}_{b}$; let $\mathfrak{B}_{b}$ be the space of all $H$-valued stochastic processes $x(t),-\infty<t \leq b$, such that $x_{0}=\phi \in \mathfrak{B}_{h}$, let $\|\cdot\|$ be a seminorm in $\mathfrak{B}_{b}$ defined by

$$
\|x\|_{b}=\left\|x_{0}\right\|_{\mathfrak{B}_{h}}+\sup _{s \in[0, b]}\left(E|x(s)|^{2}\right)^{1 / 2}, \quad x \in \mathfrak{B}_{b} .
$$

Lemma 7 (see [27]). Suppose $x \in \mathfrak{B}_{b}$; then for $t \in J, x_{t} \in \mathfrak{B}_{h}$. Moreover

$$
l E^{1 / 2}|x(t)|^{2} \leq\left\|x_{t}\right\|_{\mathfrak{B}_{h}} \leq l \sup _{s \in[0, t]}\left(E|x(s)|^{2}\right)^{1 / 2}+\left\|x_{0}\right\|_{\mathfrak{B}_{h}},
$$

where $l=\int_{-\infty}^{0} h(s) d s<+\infty$.

Now, consider the multivalued map $\mathfrak{L}: \mathfrak{B}_{b} \rightarrow 2^{\mathfrak{B}_{b}}$ defined by $\mathbb{Q} x$ the set of $\rho \in \mathfrak{B}_{b}$ such that

$$
\rho(t)=\left\{\begin{array}{l}
\phi(t) \\
\quad t \in(-\infty, 0] \\
T(t)[\phi(0)-f(0, \phi)]+f\left(t, x_{t}\right) \\
\quad+\int_{0}^{t} A T(t-s) f\left(s, x_{s}\right) d s \\
+\int_{0}^{t} T(t-s) g(s) d w(s) \\
+\int_{0}^{t} T(t-\eta) B W^{-1} \\
\quad+\left\{x_{1}-T(b)(\phi(0)-f(0, \phi))-f\left(b, x_{b}\right)\right. \\
\quad-\int_{0}^{b} A T(b-s) f\left(s, x_{s}\right) d s \\
\left.\quad-\int_{0}^{b} T(b-s) g(s) d w(s)\right\}(\eta) d \eta, \\
\end{array}\right.
$$

where $g \in S_{G, x}$.
We will show that the operator $\mathfrak{Q}$ has fixed-points, which are then a solution of system (1). For $\phi \in \mathfrak{B}_{h}$, we define $\bar{\phi}$ by

$$
\bar{\phi}(t)= \begin{cases}\phi(t), & -\infty<t \leq 0 \\ T(t) \phi(0), & 0 \leq t \leq b\end{cases}
$$

then $\bar{\phi} \in \mathfrak{B}_{b}$. Set

$$
x(t)=y(t)+\bar{\phi}(t), \quad-\infty<t \leq b .
$$

It is clear to see that $x$ satisfies (7) if and only if $y$ satisfies $y_{0}=0$ and

$$
\begin{aligned}
y(t)= & -T(t) f(0, \phi)+f\left(t, y_{t}+\bar{\phi}_{t}\right) \\
& +\int_{0}^{t} A T(t-s) f\left(s, y_{s}+\bar{\phi}_{s}\right) d s \\
& +\int_{0}^{t} T(t-s) g(s) d w(s)+\int_{0}^{t} T(t-\eta) B W^{-1} \\
& \times\left\{x_{1}-T(b)(\phi(0)-f(0, \phi))-f\left(b, y_{b}+\bar{\phi}_{b}\right)\right. \\
& \quad-\int_{0}^{b} A T(b-s) f\left(s, y_{s}+\bar{\phi}_{s}\right) d s \\
& \left.\quad-\int_{0}^{b} T(b-s) g(s) d w(s)\right\}(\eta) d \eta, \quad t \in J .
\end{aligned}
$$

Let $\mathfrak{B}_{b}^{0}=\left\{y \in \mathfrak{B}_{b}: y_{0}=0 \in \mathfrak{B}_{h}\right\}$. For any $y \in \mathfrak{B}_{b}^{0}$,

$$
\|y\|_{b}=\left\|y_{0}\right\|_{\mathfrak{B}_{h}}+\sup _{s \in[0, b]} E^{1 / 2}|y(s)|^{2}=\sup _{s \in[0, b]} E^{1 / 2}|y(s)|^{2} .
$$

Thus $\left(\mathfrak{B}_{b}^{0},\|\cdot\|_{b}\right)$ is a Banach space. Set $\mathfrak{B}_{q}=\left\{y \in \mathfrak{B}_{b}^{0}:\|y\|_{b} \leq\right.$ $q$ \} for some $q \geq 0$; then $\mathfrak{B}_{q} \subseteq \mathfrak{B}_{b}^{0}$ is uniformly bounded, for any $y \in \mathfrak{B}_{q}$; from Lemma 7 , we have

$$
\begin{aligned}
\left\|y_{t}+\bar{\phi}_{t}\right\|_{\mathfrak{B}_{h}} \leq & \left\|y_{t}\right\|_{\mathfrak{B}_{h}}+\left\|\bar{\phi}_{t}\right\|_{\mathfrak{B}_{h}} \\
\leq & l \sup _{0 \leq s \leq t} E^{1 / 2}|y(s)|^{2}+\left\|y_{0}\right\|_{\mathfrak{B}_{h}} \\
& \quad+l \sup _{0 \leq s \leq t} E^{1 / 2}|\bar{\phi}(s)|^{2}+\left\|\bar{\phi}_{0}\right\|_{\mathfrak{B}_{h}} \\
\leq & l q+\|\phi\|_{\mathfrak{B}_{h}}+l \sup _{0 \leq s \leq t}|T(s)| E^{1 / 2}|\phi(0)|^{2}
\end{aligned}
$$

$$
\leq l\left(q+M_{1} E^{1 / 2}|\phi(0)|^{2}\right)+\|\phi\|_{\mathfrak{B}_{h}}=q^{\prime} .
$$


Define the multivalued map $\mathfrak{\Omega}_{1}: \mathfrak{B}_{b}^{0} \rightarrow 2^{\mathfrak{B}_{b}^{0}}$ defined by $\mathfrak{\Omega}_{1} y$ the set of $\bar{\rho} \in \mathfrak{B}_{b}^{0}$ such that

$$
\bar{\rho}(t)=\left\{\begin{array}{c}
0, \\
\quad t \in(-\infty, 0], \\
-T(t) f(0, \phi)+f\left(t, y_{t}+\bar{\phi}_{t}\right) \\
\quad+\int_{0}^{t} A T(t-s) f\left(s, y_{s}+\bar{\phi}_{s}\right) d s \\
\quad+\int_{0}^{t} T(t-s) g(s) d w(s) \\
+\int_{0}^{t} T(t-\eta) B W^{-1} \\
\quad \times \quad x_{1}-T(b)(\phi(0)-f(0, \phi))-f\left(b, y_{b}+\bar{\phi}_{b}\right) \\
\quad-\int_{0}^{b} A T(b-s) f\left(s, y_{s}+\bar{\phi}_{s}\right) d s \\
\left.\quad-\int_{0}^{b} T(b-s) g(s) d w(s)\right\}(\eta) d \eta, \\
\end{array}\right.
$$

Theorem 8. Assume that hypotheses $\left(H_{1}\right)-\left(H_{7}\right)$ hold; then system (1) is controllable on J.

Proof. We divide the proof into several steps.

Step 1. We show that there exists an open set $U$ and there does not exist $y \in \partial U$, for some $\lambda \in(0,1)$, such that $y \in \lambda \mathbb{\Omega}_{1}(y)$.

Let $\lambda \in(0,1)$ and let $y \in \lambda \mathfrak{\Omega}_{1} y$. Then there exists $g \in S_{G, y}$ such that

$$
\begin{aligned}
y(t)= & -\lambda T(t) f(0, \phi)+\lambda f\left(t, y_{t}+\bar{\phi}_{t}\right) \\
& +\lambda \int_{0}^{t} A T(t-s) f\left(s, y_{s}+\bar{\phi}_{s}\right) d s \\
& +\lambda \int_{0}^{t} T(t-s) g(s) d w(s)+\lambda \int_{0}^{t} T(t-\eta) B W^{-1} \\
& \times\left\{x_{1}-T(b)(\phi(0)-f(0, \phi))-f\left(b, y_{b}+\bar{\phi}_{b}\right)\right. \\
& \quad-\int_{0}^{b} A T(b-s) f\left(s, y_{s}+\bar{\phi}_{s}\right) d s \\
& \left.\quad-\int_{0}^{b} T(b-s) g(s) d w(s)\right\}(\eta) d \eta, \quad t \in J .
\end{aligned}
$$$$
E|y(t)|^{2} \leq 5 M_{1}^{2} E|f(0, \phi)|^{2}+5 E\left|f\left(t, y_{t}+\phi_{t}\right)\right|^{2}
$$$$
+5 b \int_{0}^{t}\left[\frac{c_{1-\beta}}{(t-s)^{1-\beta}}\right]^{2}\left(c_{1}\left\|y_{s}+\bar{\phi}_{s}\right\|_{\mathfrak{B}_{h}}^{2}+c_{2}\right) d s
$$$$
+5 M_{1}^{2} \int_{0}^{t} p(s) \psi\left(\left\|y_{s}+\bar{\phi}_{s}\right\|_{\mathfrak{B}_{h}}^{2}\right) d s
$$$$
+25 b^{2} M_{1}^{2} M_{3}^{2} M_{4}^{2}
$$

$$
\begin{aligned}
& \times\left\{E\left|x_{1}\right|^{2}+2 M_{1}^{2} E|\phi(0)|^{2}\right. \\
& +2 M_{1}^{2}\left\|(-A)^{-\beta}\right\|^{2}\left(c_{1}\|\phi\|_{\mathfrak{B}_{h}}^{2}+c_{2}\right) \\
& +\left\|(-A)^{-\beta}\right\|^{2}\left(c_{1}\left\|y_{t}+\bar{\phi}_{t}\right\|_{\mathfrak{B}_{h}}^{2}+c_{2}\right) \\
& +\int_{0}^{b}\left[\frac{c_{1-\beta}}{(b-s)^{1-\beta}}\right]^{2}\left(c_{1}\left\|y_{s}+\bar{\phi}_{s}\right\|_{\mathfrak{B}_{h}}^{2}+c_{2}\right) d s \\
& \left.+M_{1}^{2} \int_{0}^{b} p(s) \psi\left(\left\|y_{s}+\bar{\phi}_{s}\right\|_{\mathfrak{B}_{h}}^{2}\right) d s\right\} \\
& \leq 5 M_{1}^{2}\left\|(-A)^{-\beta}\right\|^{2}\left(c_{1}\|\phi\|_{\mathfrak{B}_{h}}^{2}+c_{2}\right) \\
& +5\left\|(-A)^{-\beta}\right\|^{2}\left(c_{1}\left\|y_{t}+\bar{\phi}_{t}\right\|_{\mathfrak{B}_{h}}^{2}+c_{2}\right) \\
& +\frac{5 b^{2 \beta} c_{2} c_{1-\beta}^{2}}{2 \beta-1}+5 b c_{1} c_{1-\beta}^{2} \int_{0}^{t} \frac{\left\|y_{s}+\bar{\phi}_{s}\right\|_{\mathfrak{B}_{h}}^{2}}{(t-s)^{2-2 \beta}} d s \\
& +5 M_{1}^{2} \int_{0}^{t} p(s) \psi\left(\left\|y_{s}+\bar{\phi}_{s}\right\|_{\mathfrak{B}_{h}}^{2}\right) d s \\
& +25 b^{2} M_{1}^{2} M_{3}^{2} M_{4}^{2} \\
& \times\left\{E\left|x_{1}\right|^{2}+2 M_{1}^{2} E|\phi(0)|^{2}\right. \\
& +2 M_{1}^{2}\left\|(-A)^{-\beta}\right\|^{2}\left(c_{1}\|\phi\|_{\mathfrak{B}_{h}}^{2}+c_{2}\right) \\
& +\left\|(-A)^{-\beta}\right\|^{2}\left(c_{1}\left\|y_{t}+\bar{\phi}_{t}\right\|_{\mathfrak{B}_{h}}^{2}+c_{2}\right) \\
& +\frac{b^{2 \beta-1} c_{2} c_{1-\beta}^{2}}{2 \beta-1}+c_{1} c_{1-\beta}^{2} \int_{0}^{b} \frac{\left\|y_{s}+\bar{\phi}_{s}\right\|_{\mathfrak{B}_{h}}^{2}}{(b-s)^{2-2 \beta}} d s \\
& \left.+M_{1}^{2} \int_{0}^{b} p(s) \psi\left(\left\|y_{s}+\bar{\phi}_{s}\right\|_{\mathfrak{B}_{h}}^{2}\right) d s\right\} .
\end{aligned}
$$

Since

$$
\begin{gathered}
\left\|y_{t}+\bar{\phi}_{t}\right\|_{\mathfrak{B}_{h}} \leq l \sup _{0 \leq s \leq t} E^{1 / 2}|y(s)|^{2}+\|\phi\|_{\mathfrak{B}_{h}}+l M_{1} E^{1 / 2}|\phi(0)|^{2}, \\
\|y\|_{b}=\sup _{t \in[0, b]}\left\{E|y(t)|^{2}\right\}^{1 / 2} \\
\leq\left\{5 M_{1}^{2}\left\|(-A)^{-\beta}\right\|^{2}\left(c_{1}\|\phi\|_{\mathfrak{B}_{h}}^{2}+c_{2}\right)\right. \\
+5\left\|(-A)^{-\beta}\right\|^{2} c_{2}+\frac{5 b^{2 \beta} c_{2} c_{1-\beta}^{2}}{2 \beta-1} \\
+25 b^{2} M_{1}^{2} M_{3}^{2} M_{4}^{2}
\end{gathered}
$$




$$
\begin{aligned}
& \times\left[E\left|x_{1}\right|^{2}+2 M_{1}^{2} E|\phi(0)|^{2}\right. \\
& +2 M_{1}^{2}\left\|(-A)^{-\beta}\right\|^{2}\left(c_{1}\|\phi\|_{\mathfrak{B}_{h}}^{2}+c_{2}\right) \\
& \left.+\left\|(-A)^{-\beta}\right\|^{2} c_{2}+\frac{b^{2 \beta-1} c_{2} c_{1-\beta}^{2}}{2 \beta-1}\right] \\
& +\left[15\left\|(-A)^{-\beta}\right\|^{2} c_{1}+75 b^{2} M_{1}^{2} M_{3}^{2} M_{4}^{2}\left\|(-A)^{-\beta}\right\|^{2} c_{1}\right] \\
& \times\left[l^{2} \sup _{0 \leq s \leq b} E|y(s)|^{2}+\|\phi\|_{\mathfrak{B}_{h}}^{2}+l^{2} M_{1}^{2} E|\phi(0)|^{2}\right] \\
& +\left[15 b c_{1} c_{1-\beta}^{2}+75 b^{2} M_{1}^{2} M_{3}^{2} M_{4}^{2}\right] \\
& \times \int_{0}^{b} \frac{l^{2} \sup _{0 \leq s \leq b} E|y(s)|^{2}+\|\phi\|_{\mathfrak{B}_{h}}^{2}+l^{2} M_{1}^{2} E|\phi(0)|^{2}}{(b-s)^{2-2 \beta}} \\
& +\left[5 M_{1}^{2}+25 b^{2}\left(M_{1} M_{2} M_{3} M_{4}\right)^{2}\right] \\
& \times \int_{0}^{b} p(s) \psi\left(3 l^{2} \sup _{0 \leq s \leq b} E|y(s)|^{2}+3\|\phi\|_{\mathfrak{B}_{h}}^{2}\right. \\
& \left.\left.+3 l^{2} M_{1}^{2} E|\phi(0)|^{2}\right) d s\right\}^{1 / 2} \\
& =\left\{F+k_{1}\left(l^{2} \sup _{0 \leq s \leq b} E|y(s)|^{2}+q^{*}\right)\right. \\
& +k_{2} \int_{0}^{b} \frac{l^{2} \sup _{0 \leq s \leq b} E|y(s)|^{2}+q^{*}}{(b-s)^{2-2 \beta}} d s \\
& \left.+k_{3} \int_{0}^{b} p(s) \psi\left(3 l^{2} \sup _{0 \leq s \leq b} E|y(s)|^{2}+3 q^{*}\right) d s\right\}^{1 / 2}
\end{aligned}
$$

where $F, k_{1}, k_{2}, k_{3}$, and $q^{*}$ are defined in $H_{5}$.

Consequently

$$
\begin{aligned}
\|y\|_{b}^{2} \leq & F+k_{1} q^{*}+k_{1} l^{2}\|y\|_{b}^{2}+\frac{k_{2} b^{2 \beta-1}}{2 \beta-1}\left(l^{2}\|y\|_{b}^{2}+q^{*}\right) \\
& +k_{3} \psi\left(3 l^{2}\|y\|_{b}^{2}+3 q^{*}\right) \int_{0}^{b} p(s) d s \\
= & F^{*}+\bar{M}\|y\|_{b}^{2}+k_{3} \psi\left(3 l^{2}\|y\|_{b}^{2}+3 q^{*}\right) \int_{0}^{b} p(s) d s .
\end{aligned}
$$

So

$$
\frac{\|y\|_{b}^{2}}{F^{*}+\bar{M}\|y\|_{b}^{2}+k_{3} \psi\left(3 l^{2}\|y\|_{b}^{2}+3 q^{*}\right) \int_{0}^{b} p(s) d s} \leq 1 .
$$

Then by $H_{5}$ there exists $r$ such that $\|y\|_{b}^{2} \neq r$; that is, $\|y\|_{b} \neq r^{1 / 2}$. Define the set: $U=\left\{z \in \mathscr{B}_{b}^{0}:\|z\|_{b}<r^{1 / 2}\right\}$. From the choice of $U$, we know that there does not exist $y \in \partial U$, for some $\lambda \in(0,1)$, such that $y \in \lambda \mathfrak{Q}_{1}(y)$.

Step 2. $\mathfrak{\Omega}_{1} y$ is convex for each $y \in \mathfrak{B}_{b}^{0}$.

In fact, if $\bar{\rho}_{1}, \bar{\rho}_{2}$ belong to $\mathfrak{Q}_{1} y$, then there exist $g_{1}, g_{2} \in$ $S_{G, y}$ such that for each $t \in J$ we have

$$
\begin{aligned}
\bar{\rho}_{i}(t)= & -T(t) f(0, \phi)+f\left(t, y_{t}+\bar{\phi}_{t}\right) \\
& +\int_{0}^{t} A T(t-s) f\left(s, y_{s}+\bar{\phi}_{s}\right) d s \\
& +\int_{0}^{t} T(t-s) g_{i}(s) d w(s) \\
& +\int_{0}^{t} T(t-\eta) B W^{-1} \\
& \times\left\{x_{1}-T(b)(\phi(0)-f(0, \phi))-f\left(b, y_{b}+\bar{\phi}_{b}\right)\right. \\
& \quad-\int_{0}^{b} A T(b-s) f\left(s, y_{s}+\bar{\phi}_{s}\right) d s \\
& \left.\quad-\int_{0}^{b} T(b-s) g_{i}(s) d w(s)\right\}(\eta) d \eta, \quad t \in J .
\end{aligned}
$$

Let $\lambda \in[0,1]$; we have

$$
\begin{aligned}
&\left(\lambda \bar{\rho}_{1}\right.\left.+(1-\lambda) \bar{\rho}_{2}\right)(t) \\
&=-T(t) f(0, \phi)+f\left(t, y_{t}+\bar{\phi}_{t}\right) \\
&+\int_{0}^{t} A T(t-s) f\left(s, y_{s}+\bar{\phi}_{s}\right) d s \\
&+\int_{0}^{t} T(t-s)\left[\lambda g_{1}(s)+(1-\lambda) g_{2}(s)\right] d w(s) \\
&+\int_{0}^{t} T(t-\eta) B W^{-1} \\
& \times\left\{x_{1}-T(b)(\phi(0)-f(0, \phi))\right. \\
& \quad-f\left(b, y_{b}+\bar{\phi}_{b}\right)-\int_{0}^{b} A T(b-s) f\left(s, y_{s}+\bar{\phi}_{s}\right) d s \\
&\left.\quad-\int_{0}^{b} T(b-s)\left[\lambda g_{1}(s)+(1-\lambda) g_{2}(s)\right] d w(s)\right\}(\eta) d \eta .
\end{aligned}
$$

Since $S_{G, y}$ is convex, we have $\lambda \bar{\rho}_{1}+(1-\lambda) \bar{\rho}_{2} \in \mathfrak{\Omega}_{1} y$.

Step 3. $\mathfrak{\Omega}_{1}$ maps bounded set into bounded set in $\mathfrak{B}_{b}^{0}$. Indeed, it is enough to show that there exists a positive constant $\Lambda$ such that for each $\bar{\rho} \in \mathfrak{R}_{1} y, y \in \mathfrak{B}_{q}=\left\{y \in \mathfrak{B}_{b}^{0}:\|y\|_{b} \leq q\right\}$ 
one has $\|\bar{\rho}\|_{b} \leq \Lambda$. If $\bar{\rho} \in \mathfrak{Q}_{1} y$, then there exist $g \in S_{G, y}$, such that for each $t \in J$,

$$
\begin{aligned}
\bar{\rho}(t)= & -T(t) f(0, \phi)+f\left(t, y_{t}+\bar{\phi}_{t}\right) \\
& +\int_{0}^{t} A T(t-s) f\left(s, y_{s}+\bar{\phi}_{s}\right) d s \\
& +\int_{0}^{t} T(t-s) g(s) d w(s) \\
& +\int_{0}^{t} T(t-\eta) B W^{-1} \\
& \times\left\{x_{1}-T(b)(\phi(0)-f(0, \phi))-f\left(b, x_{b}\right)\right. \\
& \quad-\int_{0}^{b} A T(b-s) f\left(s, y_{s}+\bar{\phi}_{s}\right) d s \\
& \left.\quad-\int_{0}^{b} T(b-s) g(s) d w(s)\right\}(\eta) d \eta .
\end{aligned}
$$

So, from Lemma 1(c) for $t \in J$

$$
\begin{aligned}
& E|\bar{\rho}(t)|^{2} \\
& =E \mid-T(t) f(0, \phi)+f\left(t, y_{t}+\bar{\phi}_{t}\right) \\
& +\quad \int_{0}^{t} A T(t-s) f\left(s, y_{s}+\bar{\phi}_{s}\right) d s \\
& +\int_{0}^{t} T(t-s) g(s) d w(s) \\
& +\int_{0}^{t} T(t-\eta) B W^{-1} \\
& \quad \times\left\{x_{1}-T(b)(\phi(0)-f(0, \phi))-f\left(b, x_{b}\right)\right. \\
& \quad-\int_{0}^{b} A T(b-s) f\left(s, y-s+\bar{\phi}_{s}\right) d s \\
& \left.\quad-\int_{0}^{b} T(b-s) g(s) d w(s)\right\}\left.(\eta) d \eta\right|^{2} \\
& +5 E|-T(t) f(0, \phi)|^{2}+5 E\left|f\left(t, y_{t}+\bar{\phi}_{t}\right)\right|^{2} \\
& +5 E\left|\int_{0}^{t} A T(t-s) f\left(s, y_{s}+\bar{\phi}_{s}\right) d s\right|^{2} \\
& +5 E\left\|\int_{0}^{t} T(t-s) g(s) d w(s)\right\|_{2}^{2} \\
& +5 E \mid \int_{0}^{t} T(t-\eta) B W^{-1}
\end{aligned}
$$

$$
\begin{aligned}
& \times\left\{x_{1}-T(b)(\phi(0)-f(0, \phi))-f\left(b, x_{b}\right)\right. \\
& -\int_{0}^{b} A T(b-s) f\left(s, y_{s}+\bar{\phi}_{s}\right) d s \\
& \left.-\int_{0}^{b} T(b-s) g(s) d w(s)\right\}\left.(\eta) d \eta\right|^{2} \\
& \leq 5 M_{1}^{2}\left\|(-A)^{-\beta}\right\|^{2} E\left|(-A)^{\beta} f(0, \phi)\right|^{2} \\
& +5\left\|(-A)^{-\beta}\right\|^{2} E\left|(-A)^{\beta} f\left(t, y_{t}+\bar{\phi}_{t}\right)\right|^{2} \\
& +5 E\left(\int_{0}^{t}\left|(-A)^{1-\beta} T(t-s)\right|\left|(-A)^{\beta} f\left(s, y-s+\bar{\phi}_{s}\right)\right| d s\right)^{2} \\
& +5 \int_{0}^{t} E\|T(t-s) g(s)\|_{2}^{2} d s \\
& +5 b \int_{0}^{t} E \mid T(t-\eta) B W^{-1} \\
& \times\left\{x_{1}-T(b)(\phi(0)-f(0, \phi))-f\left(b, y_{b}+\bar{\phi}_{b}\right)\right. \\
& -\int_{0}^{b} A T(b-s) f\left(s, y_{s}+\bar{\phi}_{s}\right) d s \\
& \left.-\int_{0}^{b} T(b-s) g(s) d w(s)\right\}\left.\right|^{2}(\eta) d \eta \\
& \leq 5 M_{1}^{2}\left\|(-A)^{-\beta}\right\|^{2}\left(c_{1}\|\phi\|^{2}+c_{2}\right)+5\left\|(-A)^{-\beta}\right\|^{2}\left(c_{1} q^{\prime 2}+c_{2}\right) \\
& +5 \int_{0}^{t}\left|(-A)^{1-\beta} T(t-s)\right|^{2} d s \int_{0}^{t} E\left|(-A)^{\beta} f\left(s, y_{s}+\bar{\phi}_{s}\right)\right|^{2} d s \\
& +5 M_{1}^{2} \sup _{x \in\left[0, q^{\prime}\right]} \psi\left(x^{2}\right) \int_{0}^{b} p(s) d s+5 b M_{1}^{2} M_{3}^{2} M_{4}^{2} \\
& \times \int_{0}^{t} E \mid x_{1}-T(b)(\phi(0)-f(0, \phi))-f\left(b, y_{b}+\bar{\phi}_{b}\right) \\
& -\int_{0}^{b} A T(b-s) f\left(s, y_{s}+\bar{\phi}_{s}\right) d s \\
& -\left.\int_{0}^{b} T(b-s) g(s) d w(s)\right|^{2}(\eta) d \eta \\
& \leq 5 M_{1}^{2}\left\|(-A)^{-\beta}\right\|^{2}\left(c_{1}\|\phi\|^{2}+c_{2}\right)+5\left\|(-A)^{-\beta}\right\|^{2}\left(c_{1} q^{\prime 2}+c_{2}\right) \\
& +5 \int_{0}^{t}\left(\frac{c_{1-\beta}}{(t-s)^{1-\beta}}\right)^{2} d s \int_{0}^{t}\left(c_{1}\left\|y_{s}+\bar{\phi}_{s}\right\|^{2}+c_{2}\right) d s \\
& +5 M_{1}^{2} \sup _{x \in\left[0, q^{\prime}\right]} \psi\left(x^{2}\right) \int_{0}^{b} p(s) d s+5 b M_{1}^{2} M_{3}^{2} M_{4}^{2} \\
& \times \int_{0}^{t}\left(5 E\left|x_{1}\right|^{2}+5 M_{1}^{2} E|\phi(0)-f(0, \phi)|^{2}\right.
\end{aligned}
$$




$$
\begin{aligned}
& +5 E\left|f\left(b, y_{b}+\bar{\phi}_{b}\right)\right|^{2} \\
& +5 E\left|\int_{0}^{b} A T(b-s) f\left(s, y_{s}+\bar{\phi}_{s}\right) d s\right|^{2} \\
& \left.+5 E\left\|\int_{0}^{b} T(b-s) g(s) d w(s)\right\|_{2}^{2}\right)(\eta) d \eta \\
& \leq 5 M_{1}^{2}\left\|(-A)^{-\beta}\right\|^{2}\left(c_{1}\|\phi\|^{2}+c_{2}\right) \\
& +5\left\|(-A)^{-\beta}\right\|^{2}\left(c_{1} q^{\prime 2}+c_{2}\right)+5 c_{1-\beta}^{2} \frac{1}{2 \beta-1} b^{2 \beta}\left(c_{1} q^{\prime 2}+c_{2}\right) \\
& +5 M_{1}^{2} \sup _{x \in\left[0, q^{\prime}\right]} \psi\left(x^{2}\right) \int_{0}^{b} p(s) d s+5 b M_{1}^{2} M_{3}^{2} M_{4}^{2} \\
& \times \int_{0}^{t}\left\{5\left|x_{1}\right|^{2}+5 M_{1}^{2}\left(2 E|\phi(0)|^{2}+2\left\|(-A)^{-\beta}\right\|^{2}\left(c_{1}\|\phi\|^{2}+c_{2}\right)\right)\right. \\
& +5\left\|(-A)^{-\beta}\right\|^{2}\left(c_{1}\left\|y_{b}+\bar{\phi}_{b}\right\|^{2}+c_{2}\right) \\
& +5 \int_{0}^{b}\left|(-A)^{1-\beta} T(b-s)\right|^{2} d s \\
& \times \int_{0}^{b} E\left|(-A)^{\beta} f\left(s, y_{s}+\bar{\phi}_{s}\right) d s\right|^{2} d s \\
& \left.+5 \int_{0}^{b} E\|T(b-s) g(s)\|_{2}^{2}\right\}(\eta) d \eta \\
& \leq 5 M_{1}^{2}\left\|(-A)^{-\beta}\right\|^{2}\left(c_{1}\|\phi\|^{2}+c_{2}\right) \\
& +5\left\|(-A)^{-\beta}\right\|^{2}\left(c_{1} q^{\prime 2}+c_{2}\right)+5 \frac{c_{1-\beta}^{2} b^{2 \beta}}{2 \beta-1}\left(c_{1} q^{\prime 2}+c_{2}\right) \\
& +5 M_{1}^{2} \sup _{x \in\left[0, q^{\prime}\right]} \psi\left(x^{2}\right) \int_{0}^{b} p(s) d s+5 b M_{1}^{2} M_{3}^{2} M_{4}^{2} \\
& \times \int_{0}^{t}\left\{5\left|x_{1}\right|^{2}+5 M_{1}^{2}\left(2 E|\phi(0)|^{2}+2\left\|(-A)^{-\beta}\right\|^{2}\left(c_{1}\|\phi\|^{2}+c_{2}\right)\right)\right. \\
& +5\left\|(-A)^{-\beta}\right\|^{2}\left(c_{1} q^{\prime 2}+c_{2}\right) \\
& +5 \int_{0}^{b}\left(\frac{c_{1-\beta}}{(b-s)^{1-\beta}}\right)^{2} d s \int_{0}^{b}\left(c_{1}\left\|y_{s}+\bar{\phi}_{s}\right\|^{2}+c_{2}\right) d s \\
& \left.+5 M_{1}^{2} \sup _{x \in\left[0, q^{\prime}\right]} \psi\left(x^{2}\right) \int_{0}^{b} p(s) d s\right\}(\eta) d \eta \\
& \leq 5 M_{1}^{2}\left\|(-A)^{-\beta}\right\|^{2}\left(c_{1}\|\phi\|^{2}+c_{2}\right) \\
& +5\left\|(-A)^{-\beta}\right\|^{2}\left(c_{1} q^{\prime 2}+c_{2}\right)+5 \frac{c_{1-\beta}^{2} b^{2 \beta}}{2 \beta-1}\left(c_{1} q^{\prime 2}+c_{2}\right) \\
& +5 M_{1}^{2} \sup _{x \in\left[0, q^{\prime}\right]} \psi\left(x^{2}\right) \int_{0}^{b} p(s) d s+5 b^{2} M_{1}^{2} M_{3}^{2} M_{4}^{2}
\end{aligned}
$$

$$
\begin{aligned}
& \times\left\{5 E\left|x_{1}\right|^{2}+5 M_{1}^{2}\left(2 E|\phi(0)|^{2}+2\left\|(-A)^{-\beta}\right\|^{2}\left(c_{1}\|\phi\|^{2}+c_{2}\right)\right)\right. \\
& +5\left\|(-A)^{-\beta}\right\|^{2}\left(c_{1} q^{\prime 2}+c_{2}\right)+5 \frac{c_{1-\beta}^{2} b^{2 \beta}}{2 \beta-1}\left(c_{1} q^{\prime 2}+c_{2}\right) \\
& \left.+5 M_{1}^{2} \sup _{x \in\left[0, q^{\prime}\right]} \psi\left(x^{2}\right) \int_{0}^{b} p(s) d s\right\}
\end{aligned}
$$$$
=\Lambda^{2}
$$

then for each $\bar{\rho} \in L_{1}\left(\mathfrak{B}_{q}\right)$, we have

$$
\|\bar{\rho}\|_{b} \leq \Lambda
$$

Step 4. $\mathbb{\Omega}_{1}$ has a closed graph.

Let $y^{(n)} \rightarrow y^{*}, \bar{\rho}_{n} \in \mathfrak{R}_{1} y^{(n)}$, and $\bar{\rho}_{n} \rightarrow \bar{\rho}_{*}$. We will prove that $\bar{\rho}_{*} \in \mathfrak{Q}_{1} y^{*}$. Indeed, $\bar{\rho}_{n} \in \mathfrak{\Omega}_{1} y^{(n)}$ means that there exists $g_{n} \in S_{G, y^{(n)}}$, such that

$$
\begin{aligned}
\bar{\rho}_{n}(t)= & -T(t) f(0, \phi)+f\left(t, y_{t}^{(n)}+\bar{\phi}_{t}\right) \\
& +\int_{0}^{t} A T(t-s) f\left(s, y_{s}^{(n)}+\bar{\phi}_{s}\right) d s \\
& +\int_{0}^{t} T(t-s) g_{n}(s) d w(s) \\
& +\int_{0}^{t} T(t-\eta)\left(B u_{y^{(n)}}(\eta)\right) d \eta, \quad t \in J,
\end{aligned}
$$

where

$$
\begin{aligned}
& u_{y(n)}(t)=W^{-1}\left\{x_{1}-T(b)(\phi(0)-f(0, \phi))\right. \\
&-f\left(b, y_{b}+\bar{\phi}_{b}\right) \\
&-\int_{0}^{b} A T(b-s) f\left(s, y_{s}+\bar{\phi}_{s}\right) d s \\
&\left.-\int_{0}^{b} T(b-s) g_{n}(s) d w(s)\right\}(t) .
\end{aligned}
$$

We must prove that there exists $g_{*} \in S_{G, y^{*}}$ such that

$$
\begin{aligned}
\bar{\rho}_{*}(t)= & -T(t) f(0, \phi)+f\left(t, y_{t}^{*}+\bar{\phi}_{t}\right) \\
& +\int_{0}^{t} A T(t-s) f\left(s, y_{s}^{*}+\bar{\phi}_{s}\right) d s \\
& +\int_{0}^{t} T(t-s) g_{*}(s) d w(s) \\
& +\int_{0}^{t} T(t-\eta)\left(B u_{y^{*}}(\eta)\right) d \eta, \quad t \in J,
\end{aligned}
$$


where

$$
\begin{gathered}
u_{y^{*}}(t)=W^{-1}\left\{x_{1}-T(b)(\phi(0)-f(0, \phi))-f\left(b, y_{b}^{*}+\bar{\phi}_{b}\right)\right. \\
-\int_{0}^{b} A T(b-s) f\left(s, y_{s}^{*}+\bar{\phi}_{s}\right) d s \\
\left.-\int_{0}^{b} T(b-s) g_{*}(s) d w(s)\right\}(t) .
\end{gathered}
$$

Set

$$
\begin{gathered}
\bar{u}_{y}(t)=W^{-1}\left\{x_{1}-T(b)(\phi(0)-f(0, \phi))-f\left(b, y_{b}+\bar{\phi}_{b}\right)\right. \\
\left.-\int_{0}^{b} A T(b-s) f\left(s, y_{s}+\bar{\phi}_{s}\right) d s\right\}(t) .
\end{gathered}
$$

Since $f, W^{-1}$ are continuous, then $\bar{u}_{y^{(n)}}(t) \rightarrow \bar{u}_{y^{*}}(t)$ for $t \in J$,

$$
\begin{aligned}
& \|\left\{\bar{\rho}_{n}(t)+T(t) f(0, \phi)-f\left(t, y_{t}^{(n)}+\bar{\phi}_{t}\right)\right. \\
& \quad-\int_{0}^{t} A T(t-s) f\left(s, y_{s}^{(n)}+\bar{\phi}_{s}\right) d s \\
& \quad-\int_{0}^{t} T(t-\eta)\left(B u_{y^{(n)}}(\eta) d \eta\right\} \\
& -\left\{\bar{\rho}_{*}(t)+T(t) f(0, \phi)-f\left(t, y_{t}^{*}+\bar{\phi}_{t}\right)\right. \\
& \quad-\int_{0}^{t} A T(t-s) f\left(s, y_{s}^{*}+\bar{\phi}_{s}\right) d s \\
& \quad-\int_{0}^{t} T(t-\eta)\left(B u_{y^{*}(\eta)}(\eta) d \eta\right\} \| \longrightarrow 0, \quad \text { as } n \longrightarrow \infty .
\end{aligned}
$$

Consider the linear continuous operator

$$
\begin{aligned}
\Gamma: L^{2}(J, H) \longrightarrow C(J, H), \\
g \longrightarrow \Gamma(g)(t) \\
=\int_{0}^{t} T(t-s) g(s) d w(s) \\
\quad-\int_{0}^{t} T(t-s) B W^{-1}\left[\int_{0}^{b} T(b-\tau) g(\tau) d w(\tau)\right] d s .
\end{aligned}
$$

From Lemma 5, it follows that $T \circ S_{G}$ is a closed graph. Moreover, we have

$$
\begin{aligned}
\bar{\rho}_{n}(t) & +T(t) f(0, \phi)-f\left(t, y_{t}^{(n)}+\bar{\phi}_{t}\right) \\
& -\int_{0}^{t} A T(t-s) f\left(s, y_{s}^{(n)}+\bar{\phi}_{s}\right) d s \\
& -\int_{0}^{t} T(t-\eta) B u_{y^{(n)}}(\eta) d \eta \in \Gamma\left(S_{G, y^{(n)}}\right) .
\end{aligned}
$$

Since $y^{(n)} \rightarrow y^{*}$, it follows from Lemma 5 that

$$
\begin{aligned}
& \bar{\rho}_{*}(t)+T(t) f(0, \phi)-f\left(t, y_{t}^{*}+\bar{\phi}_{t}\right) \\
&- \int_{0}^{t} A T(t-s) f\left(s, y_{s}^{*}+\bar{\phi}_{s}\right) d s \\
&- \int_{0}^{t} T(t-\eta)\left(B \bar{u}_{y^{*}}\right)(\eta) d \eta \\
&=\int_{0}^{t} T(t-s) g_{*}(s) d w(s) \\
& \quad-\int_{0}^{t} T(t-s) B W^{-1}\left[\int_{0}^{b} T(b-\tau) g(\tau) d w(\tau)\right] d s
\end{aligned}
$$

for some $g_{*} \in S_{G, y^{*}}$.

Step 5. Multivalued function $\mathfrak{Q}_{1}: \bar{U} \rightarrow \mathscr{P}_{c p, c v}\left(\mathfrak{B}_{b}^{0}\right)$ is a condensing operator.

From the decomposition of operator $\mathfrak{\Omega}_{1}$, we define two operators $T, S$ on space $\mathfrak{B}_{b}^{0}$. For operator $T, T: \bar{U} \rightarrow$ $\mathscr{P}_{c p, c v}\left(\mathfrak{B}_{b}^{0}\right)$, for each $y, T y$ defined by the set of $h_{1}(t) \in \mathfrak{B}_{b}^{0}$ such that

$$
h_{1}(t)= \begin{cases}0, & t \in(-\infty, 0], \\ -T(t) f(0, \phi)+f\left(t, y_{t}+\bar{\phi}_{t}\right) & \\ \quad+\int_{0}^{t} A T(t-s) f\left(s, y_{s}+\bar{\phi}_{s}\right) d s & t \in J .\end{cases}
$$

For operator $S, S: \bar{U} \rightarrow \mathscr{P}_{c p, c v}\left(\mathfrak{B}_{b}^{0}\right)$, for each $y, S y$ defined by the set of $h_{2}(t) \in \mathfrak{B}_{b}^{0}$ such that

$$
h_{2}(t)=\left\{\begin{array}{c}
0, \\
\quad t \in(-\infty, 0], \\
\int_{0}^{t} T(t-s) g(s) d w(s)+\int_{0}^{t} T(t-\eta) B W^{-1} \\
\times\left\{x_{1}-T(b)(\phi(0)-f(0, \phi))-f\left(b, y_{b}+\bar{\phi}_{b}\right)\right. \\
\quad-\int_{0}^{b} A T(b-s) f\left(s, y_{s}+\bar{\phi}_{s}\right) d s \\
\left.\quad-\int_{0}^{b} T(b-s) g(s) d w(s)\right\}(\eta) d \eta, \\
t \in J .
\end{array}\right.
$$


So, operator $\mathfrak{\Omega}_{1} y=T y+S y$. Next, we will prove that operator $T$ is contraction operator and $S$ is completely continuous operator.

First, we prove operator $T$ is contractive. For each $y^{1}, y^{2} \in$ $\mathfrak{B}_{b}^{0}$, we have

$$
\begin{aligned}
& E\left|h_{1}^{1}(t)-h_{1}^{2}(t)\right|^{2} \\
& =E \mid f\left(t, y_{t}^{1}+\bar{\phi}_{t}\right)-f\left(t, y_{t}^{2}+\bar{\phi}_{t}\right) \\
& +\int_{0}^{t} A T(t-s) f\left(s, y_{s}^{1}+\bar{\phi}_{s}\right) d s \\
& -\left.\int_{0}^{t} A T(t-s) f\left(s, y_{s}^{2}+\bar{\phi}_{s}\right) d s\right|^{2} \\
& \leq 2 E\left|f\left(t, y_{t}^{1}+\bar{\phi}_{t}\right)-f\left(t, y_{t}^{2}+\bar{\phi}_{t}\right)\right|^{2} \\
& +2 E \mid \int_{0}^{t} A T(t-s) f\left(s, y_{s}^{1}+\bar{\phi}_{s}\right) d s \\
& -\left.\int_{0}^{t} A T(t-s) f\left(s, y_{s}^{2}+\bar{\phi}_{s}\right) d s\right|^{2} \\
& \leq 2\left\|(-A)^{-\beta}\right\|^{2} q^{2}\left\|y_{t}^{1}-y_{t}^{2}\right\|^{2} \\
& +2 E \mid \int_{0}^{t}(-A)^{1-\beta} T(t-s) \\
& \times\left[(-A)^{\beta} f\left(s, y_{s}^{1}+\bar{\phi}_{s}\right)\right. \\
& \left.-(-A)^{\beta} f\left(s, y_{s}^{2}+\bar{\phi}_{s}\right)\right]\left.d s\right|^{2} \\
& \leq 2\left\|(-A)^{-\beta}\right\|^{2} q^{2}\left\|y_{t}^{1}-y_{t}^{2}\right\|^{2} \\
& +2 \int_{0}^{t}\left|(-A)^{1-\beta} T(t-s)\right|^{2} d s \\
& \times \int_{0}^{t} E\left|(-A)^{\beta} f\left(s, y_{s}^{1}+\bar{\phi}_{s}\right)-(-A)^{\beta} f\left(s, y_{s}^{2}+\bar{\phi}_{s}\right)\right|^{2} d s \\
& \leq 2\left\|(-A)^{-\beta}\right\|^{2} q^{2}\left\|y_{t}^{1}-y_{t}^{2}\right\|^{2} \\
& +2 \int_{0}^{t}\left(\frac{c_{1-\beta}}{(t-s)^{1-\beta}}\right)^{2} d s \int_{0}^{t} q^{2}\left\|y_{t}^{1}-y_{t}^{2}\right\|^{2} d s \\
& \leq 2\left\|(-A)^{-\beta}\right\|^{2} q^{2}\left\|y_{t}^{1}-y_{t}^{2}\right\|^{2} \\
& +2 c_{1-\beta}^{2} \frac{1}{2 \beta-1} b^{2 \beta-1} q^{2} \sup _{s \in[0, t]}\left\|y_{t}^{1}-y_{t}^{2}\right\|^{2} \\
& \leq\left[2\left\|(-A)^{-\beta}\right\|^{2} q^{2}+2 c_{1-\beta}^{2} \frac{1}{2 \beta-1} b^{2 \beta-1} q^{2} l^{2}\right]\left\|y^{1}-y^{2}\right\|^{2} \\
& =Q\left\|y^{1}-y^{2}\right\|^{2} \text {. }
\end{aligned}
$$

\section{So operator $T$ is contraction operator.}

Next, we will prove that operator $S$ is completely continuous.

For $t_{1}, t_{2} \in J, 0<t_{1}<t_{2}<b$, if $y \in \bar{U}, h_{2} \in S y$, then there exist $f \in S_{F, y}$ such that for arbitrary $t \in[0, b]$,

$$
\begin{aligned}
h_{2}(t)=\int_{0}^{t} T(t-s) g(s) d w(s)+\int_{0}^{t} T(t-\eta) B W^{-1} \\
\quad \times\left\{x_{1}-T(b)(\phi(0)-f(0, \phi))\right. \\
\quad-f\left(b, y_{b}+\bar{\phi}_{b}\right)-\int_{0}^{b} A T(b-s) f\left(s, y_{s}+\bar{\phi}_{s}\right) d s \\
\left.\quad-\int_{0}^{b} T(b-s) g(s) d w(s)\right\}(\eta) d \eta, \quad t \in J .
\end{aligned}
$$

Thus

$$
\begin{aligned}
& E\left|h_{2}\left(t_{2}\right)-h_{2}\left(t_{1}\right)\right|^{2} \\
& =E \mid \int_{0}^{t_{2}}\left(T\left(t_{2}-s\right)-T\left(t_{1}-s\right)\right) g(s) d w(s) \\
& +\int_{t_{1}}^{t_{2}} T\left(t_{1}-s\right) g(s) d w(s) \\
& +\int_{0}^{t_{2}}\left(T\left(t_{2}-\eta\right)-T\left(t_{1}-\eta\right)\right) \\
& \times B W^{-1}\left\{x_{1}-T(b)(\phi(0)-f(0, \phi))-f\left(b, y_{b}+\bar{\phi}_{b}\right)\right. \\
& -\int_{0}^{b} A T(b-s) f\left(s, y_{s}+\bar{\phi}_{s}\right) d s \\
& \left.-\int_{0}^{b} T(b-s) g(s) d w(s)\right\}(\eta) d \eta \\
& +\int_{t_{1}}^{t_{2}} T\left(t_{1}-\eta\right) B W^{-1} \\
& \times\left\{x_{1}-T(b)(\phi(0)-f(0, \phi))\right. \\
& -f\left(b, y_{b}+\bar{\phi}_{b}\right)-\int_{0}^{b} A T(b-s) f\left(s, y_{s}+\bar{\phi}_{s}\right) d s \\
& \left.-\int_{0}^{b} T(b-s) g(s) d w(s)\right\}\left.(\eta) d \eta\right|^{2} \\
& \leq 4 E\left|\int_{0}^{t_{2}}\left(T\left(t_{2}-s\right)-T\left(t_{1}-s\right)\right) g(s) d w(s)\right|^{2} \\
& +4 E\left|\int_{t_{1}}^{t_{2}} T\left(t_{1}-s\right) g(s) d w(s)\right|^{2}
\end{aligned}
$$


Mathematical Problems in Engineering

11

$$
\begin{aligned}
& +4 E \mid \int_{0}^{t_{2}}\left(T\left(t_{2}-\eta\right)-T\left(t_{1}-\eta\right)\right) B W^{-1} \\
& \leq 4 \int_{0}^{t_{2}}\left|T\left(t_{2}-s\right)-T\left(t_{1}-s\right)\right|^{2} E\|g(s)\|_{2}^{2} d s \\
& \times\left\{x_{1}-T(b)(\phi(0)-f(0, \phi))\right. \\
& +4 \int_{t_{1}}^{t_{2}}\left|T\left(t_{1}-s\right)^{2}\right| E\|g(s)\|_{2}^{2} d s \\
& -f\left(b, y_{b}+\bar{\phi}_{b}\right) \\
& +4 b \int_{0}^{t_{2}}\left|T\left(t_{2}-\eta\right)-T\left(t_{1}-\eta\right)\right|^{2} M_{3}^{2} M_{4}^{2} \\
& -\int_{0}^{b} A T(b-s) f\left(s, y_{s}+\bar{\phi}_{s}\right) d s \\
& \times E \mid x_{1}-T(b)(\phi(0)-f(0, \phi)) \\
& \left.-\int_{0}^{b} T(b-s) g(s) d w(s)\right\}\left.(\eta) d \eta\right|^{2} \\
& -f\left(b, y_{b}+\bar{\phi}_{b}\right) \\
& -\int_{0}^{b} A T(b-s) f\left(s, y_{s}+\bar{\phi}_{s}\right) d s \\
& +4 E \mid \int_{t_{1}}^{t_{2}} T\left(t_{1}-\eta\right) B W^{-1} \\
& -\left.\int_{0}^{b} T(b-s) g(s) d w(s)\right|^{2}(\eta) d \eta \\
& \times\left\{x_{1}-T(b)(\phi(0)-f(0, \phi))\right. \\
& -f\left(b, y_{b}+\bar{\phi}_{b}\right) \\
& -\int_{0}^{b} A T(b-s) f\left(s, y_{s}+\bar{\phi}_{s}\right) d s \\
& \left.-\int_{0}^{b} T(b-s) g(s) d w(s)\right\}\left.(\eta) d \eta\right|^{2} \\
& \leq 4 E\left\|\int_{0}^{t_{2}}\left(T\left(t_{2}-s\right)-T\left(t_{1}-s\right)\right) g(s) d w(s)\right\|_{2}^{2} \\
& +4 E\left\|\int_{t_{1}}^{t_{2}} T\left(t_{1}-s\right) g(s) d w(s)\right\|_{2}^{2} \\
& +4 E \mid \int_{0}^{t_{2}}\left(T\left(t_{2}-\eta\right)-T\left(t_{1}-\eta\right)\right) B W^{-1} \\
& \times\left\{x_{1}-T(b)(\phi(0)-f(0, \phi))\right. \\
& -f\left(b, y_{b}+\bar{\phi}_{b}\right) \\
& -\int_{0}^{b} A T(b-s) f\left(s, y_{s}+\bar{\phi}_{s}\right) d s \\
& \left.-\int_{0}^{b} T(b-s) g(s) d w(s)\right\}\left.(\eta) d \eta\right|^{2} \\
& +4 E \mid \int_{t_{1}}^{t_{2}} T\left(t_{1}-\eta\right) B W^{-1} \\
& \times\left\{x_{1}-T(b)(\phi(0)-f(0, \phi))-f\left(b, y_{b}+\bar{\phi}_{b}\right)\right. \\
& -\int_{0}^{b} A T(b-s) f\left(s, y_{s}+\bar{\phi}_{s}\right) d s \\
& \left.-\int_{0}^{b} T(b-s) g(s) d w(s)\right\}\left.(\eta) d \eta\right|^{2} \\
& +4\left(t_{2}-t_{1}\right) \int_{t_{1}}^{t_{2}}\left|T\left(t_{1}-\eta\right)\right|^{2} M_{3}^{2} M_{4}^{2} \\
& \times E \mid x_{1}-T(b)(\phi(0)-f(0, \phi))-f\left(b, y_{b}+\bar{\phi}_{b}\right) \\
& -\int_{0}^{b} A T(b-s) f\left(s, y_{s}+\bar{\phi}_{s}\right) d s \\
& -\left.\int_{0}^{b} T(b-s) g(s) d w(s)\right|^{2}(\eta) d \eta \\
& \leq 4 \int_{0}^{t_{2}}\left|T\left(t_{2}-s\right)-T\left(t_{1}-s\right)\right|^{2} E\|g(s)\|_{2}^{2} d s \\
& +4 \int_{t_{1}}^{t_{2}}\left|T\left(t_{1}-s\right)\right|^{2} E\|g(s)\|_{2}^{2} d s \\
& +4 b \int_{0}^{t_{2}}\left|T\left(t_{2}-\eta\right)-T\left(t_{1}-\eta\right)\right|^{2} M_{3}^{2} M_{4}^{2} \\
& \times\left\{5 E\left|x_{1}\right|^{2}+5 M_{1}^{2}\right. \\
& \times\left(2 E|\phi(0)|^{2}+2\left\|(-A)^{-\beta}\right\|^{2}\left(c_{1}\|\phi\|_{\mathfrak{B}_{h}}^{2}+c_{2}\right)\right) \\
& +5\left\|(-A)^{-\beta}\right\|^{2}\left(c_{1}\left\|y_{b}+\bar{\phi}_{b}\right\|_{\mathfrak{B}_{h}}^{2}+c_{2}\right) \\
& +5 b \int_{0}^{b} E\left|A T(b-s) f\left(s, y_{s}+\bar{\phi}_{s}\right)\right|^{2} d s \\
& \left.+5 \int_{0}^{b} E\|T(b-s) g(s)\|_{2}^{2} d s\right\}(\eta) d \eta \\
& +4\left(t_{2}-t_{1}\right) M_{3}^{2} M_{4}^{2} \int_{t_{1}}^{t_{2}}\left|T\left(t_{1}-\eta\right)\right|^{2} \\
& \times\left\{5 E\left|x_{1}\right|^{2}+5 M_{1}^{2}\right.
\end{aligned}
$$




$$
\begin{aligned}
& \times\left(2 E|\phi(0)|^{2}+2\left\|(-A)^{-\beta}\right\|^{2}\left(c_{1}\|\phi\|_{\mathfrak{B}_{h}}^{2}+c_{2}\right)\right) \\
& +5\left\|(-A)^{-\beta}\right\|^{2}\left(c_{1}\left\|y_{b}+\bar{\phi}_{b}\right\|_{\mathfrak{B}_{h}}+c_{2}\right) \\
& +5 b \int_{0}^{b} E\left|A T(b-s) f\left(s, y_{s}+\bar{\phi}_{s}\right)\right|^{2} d s \\
& \left.+5 \int_{0}^{b} E\|T(b-s) g(s)\|_{2}^{2} d s\right\}(\eta) d \eta \\
& \leq 4 \int_{0}^{t_{2}}\left|T\left(t_{2}-s\right)-T\left(t_{1}-s\right)\right|^{2} E\|g(s)\|_{2}^{2} d s \\
& +4 \int_{t_{1}}^{t_{2}}\left|T\left(t_{1}-s\right)\right|^{2} E\|g(s)\|_{2}^{2} d s \\
& +4 b \int_{0}^{t_{2}}\left|T\left(t_{2}-\eta\right)-T\left(t_{1}-\eta\right)\right|^{2} M_{3}^{2} M_{4}^{2} \\
& \times\left\{5 E\left|x_{1}\right|^{2}+5 M_{1}^{2}\right. \\
& \times\left(2 E|\phi(0)|^{2}+2\left\|(-A)^{-\beta}\right\|^{2}\left(c_{1}\|\phi\|_{\mathfrak{B}_{h}}^{2}+c_{2}\right)\right) \\
& +5\left\|(-A)^{-\beta}\right\|^{2}\left(c_{1}\left\|y_{b}+\bar{\phi}_{b}\right\|_{\mathfrak{B}_{h}}^{2}+c_{2}\right) \\
& +5 b \int_{0}^{b}\left|(-A)^{1-\beta} T(b-s)\right|^{2} \\
& \times\left(c_{1}\left\|y_{s}+\bar{\phi}_{s}\right\|_{\mathfrak{B}_{h}}^{2}+c_{2}\right) d s \\
& \left.+5 M_{1}^{2} \int_{0}^{b} E\|g(s)\|_{2}^{2} d s\right\}(\eta) d \eta \\
& +4\left(t_{2}-t_{1}\right) M_{3}^{2} M_{4}^{2} \int_{t_{1}}^{t_{2}}\left|T\left(t_{1}-\eta\right)\right|^{2} \\
& \times\left\{5 E\left|x_{1}\right|^{2}+5 M_{1}^{2}\right. \\
& \times\left(2 E|\phi(0)|^{2}+2\left\|(-A)^{-\beta}\right\|^{2}\left(c_{1}\|\phi\|_{\mathfrak{B}_{h}}^{2}+c_{2}\right)\right) \\
& +5\left\|(-A)^{-\beta}\right\|^{2}\left(c_{1}\left\|y_{b}+\bar{\phi}_{b}\right\|_{\mathfrak{B}_{h}}^{2}+c_{2}\right) \\
& +5 b \int_{0}^{b}\left|(-A)^{1-\beta} T(b-s)\right|^{2}\left(c_{1}\left\|y_{s}+\bar{\phi}_{s}\right\|_{\mathfrak{B}_{h}}^{2}+c_{2}\right) d s \\
& \left.+5 M_{1}^{2} \int_{0}^{b} E\|g(s)\|_{2}^{2} d s\right\}(\eta) d \eta \text {. }
\end{aligned}
$$

The right-hand side of the previous inequality is independent of $y$ and tends to zero as $t_{2} \rightarrow t_{1}$. It is easy to prove that $S$ maps bounded set into bounded set, so operator $S$ is completely continuous.
Hence, it follows from Theorem 2 that the operator $\mathfrak{\Omega}_{1}$ has a fixed-point $y^{*} \in \mathfrak{B}_{b}^{0}$. Let $x(t)=y^{*}(t)+\bar{\phi}(t), t \in(-\infty, b]$. Then $x$ is a fixed-point of the operator $\mathbb{Q}$ which is a mild solution of problem (1); then system (1) is controllable on $J$.

Remark 9. Sakthivel et al. [28] considered approximate controllability for nonlinear stochastic systems with resolvent operators and unbounded delay. Approximate controllability results are being established for a class of nonlinear stochastic differential equations with resolvent operators in a real separable Hilbert spaces by using the resolvent operators and fixed-point technique.

\section{Conclusion}

In this paper, we have investigated the controllability of stochastic neutral functional differential inclusions in Hilbert spaces. Based on a fixed-point theorem with regard to condensing map, sufficient conditions for the controllability of the stochastic neutral functional differential inclusions have been derived.

\section{Acknowledgments}

This work was supported by the grant of Chongqing municipal educational commission (no. KJ120609), Natural Science Foundation Project of CSTC, 2010 BB9318 and CQ CSTC, 2009BB3057, and the Ph.D. Foundation of Chongqing Normal University under Grant no. 09XLB007.

\section{References}

[1] J. Wang and Y. Zhou, "Existence and controllability results for fractional semilinear differential inclusions," Nonlinear Analysis. Real World Applications, vol. 12, no. 6, pp. 3642-3653, 2011.

[2] J. Wang and Y. Zhou, "Complete controllability of fractional evolution systems," Communications in Nonlinear Science and Numerical Simulation, vol. 17, no. 11, pp. 4346-4355, 2012.

[3] J. Wang, Z. Fan, and Y. Zhou, "Nonlocal controllability of semilinear dynamic systems with fractional derivative in Banach spaces," Journal of Optimization Theory and Applications, vol. 154, no. 1, pp. 292-302, 2012.

[4] M. Feckan, J. Wang, and Y. Zhou, "Controllability of fractional functional evolution equations of Sobolev type via characteristic solution operators," Journal of Optimization Theory and Applications, vol. 156, no. 1, pp. 79-95, 2013.

[5] R. Sakthivel, N. I. Mahmudov, and J. J. Nieto, "Controllability for a class of fractional-order neutral evolution control systems," Applied Mathematics and Computation, vol. 218, no. 20, pp. 10334-10340, 2012.

[6] Z. Yan, "Approximate controllability of partial neutral functional differential systems of fractional order with statedependent delay," International Journal of Control, vol. 85, no. 8, pp. 1051-1062, 2012.

[7] R. Sakthivel, S. Suganya, and S. M. Anthoni, "Approximate controllability of fractional stochastic evolution equations," Computers \& Mathematics with Applications, vol. 63, no. 3, pp. 660-668, 2012. 
[8] R. Sakthivel and Y. Ren, "Complete controllability of stochastic evolution equations with jumps," Reports on Mathematical Physics, vol. 68, no. 2, pp. 163-174, 2011.

[9] R. Sakthivel, Y. Ren, and N. I. Mahmudov, "On the approximate controllability of semilinear fractional differential systems," Computers \& Mathematics with Applications, vol. 62, no. 3, pp. 1451-1459, 2011.

[10] R. Sakthivel, N. I. Mahmudov, and Y. Ren, "Approximate controllability of the nonlinear third-order dispersion equation," Applied Mathematics and Computation, vol. 217, no. 21, pp. 8507-8511, 2011.

[11] M. Benchohra, L. Górniewicz, and S. K. Ntouyas, "Controllability on infinite time horizon for first and second order functional differential inclusions in Banach spaces," Discussiones Mathematicae. Differential Inclusions, Control and Optimization, vol. 21, no. 2, pp. 261-282, 2001.

[12] J. Y. Park, Y. C. Kwun, and H. J. Lee, "Controllability of secondorder neutral functional differential inclusions in Banach spaces," Journal of Mathematical Analysis and Applications, vol. 285, no. 1, pp. 37-49, 2003.

[13] B. Liu, "Controllability of neutral functional differential and integrodifferential inclusions with infinite delay," Journal of Optimization Theory and Applications, vol. 123, no. 3, pp. 573593, 2004.

[14] B. Liu, "Controllability of impulsive neutral functional differential inclusions with infinite delay," Nonlinear Analysis. Theory, Methods \& Applications, vol. 60, no. 8, pp. 1533-1552, 2005.

[15] X. Mao, Stochastic Differential Equations and Their Applications, Horwood Publishing Series in Mathematics \& Applications, Horwood Publishing, Chichester, UK, 1997.

[16] K. Sobczyk, Stochastic Differential Equations, vol. 40 of Mathematics and Its Applications (East European Series), Kluwer Academic Publishers, Dordrecht, The Netherlands, 1991.

[17] N. I. Mahmudov, "Existence and uniqueness results for neutral SDEs in Hilbert spaces," Stochastic Analysis and Applications, vol. 24, no. 1, pp. 79-95, 2006.

[18] P. Balasubramaniam and S. K. Ntouyas, "Controllability for neutral stochastic functional differential inclusions with infinite delay in abstract space," Journal of Mathematical Analysis and Applications, vol. 324, no. 1, pp. 161-176, 2006.

[19] P. Balasubramaniam and J. P. Dauer, "Controllability of semilinear stochastic delay evolution equations in Hilbert spaces," International Journal of Mathematics and Mathematical Sciences, vol. 31, no. 3, pp. 157-166, 2002.

[20] J. Wu, Theory and Applications of Partial Functional-Differential Equations, vol. 119 of Applied Mathematical Sciences, Springer, New York, NY, USA, 1996.

[21] D. O'Regan, "Nonlinear alternatives for multivalued maps with applications to operator inclusions in abstract spaces," Proceedings of the American Mathematical Society, vol. 127, no. 12, pp. 3557-3564, 1999.

[22] K. Deimling, Multivalued Differential Equations, vol. 1 of de Gruyter Series in Nonlinear Analysis and Applications, Walter de Gruyter, Berlin, Germany, 1992.

[23] S. Hu and N. S. Papageorgiou, Handbook of Multivalued Analysis. Vol. I. Theory, vol. 419 of Mathematics and Its Applications, Kluwer Academic Publishers, Dordrecht, The Netherlands, 1997.

[24] A. Pazy, Semigroups of Linear Operators and Applications to Partial Differential Equations, vol. 44 of Applied Mathematical Sciences, Springer, New York, NY, USA, 1983.
[25] A. Lasota and Z. Opial, "An application of the Kakutani-Ky Fan theorem in the theory of ordinary differential equations," Bulletin de l'Académie Polonaise des Sciences. Série des Sciences Mathématiques, Astronomiques et Physiques, vol. 13, pp. 781-786, 1965.

[26] E. Hernández, "Existence results for partial neutral functional integrodifferential equations with unbounded delay," Journal of Mathematical Analysis and Applications, vol. 292, no. 1, pp. 194210, 2004.

[27] Y. Li and B. Liu, "Existence of solution of nonlinear neutral stochastic differential inclusions with infinite delay," Stochastic Analysis and Applications, vol. 25, no. 2, pp. 397-415, 2007.

[28] R. Sakthivel, J. J. Nieto, and N. I. Mahmudov, “Approximate controllability of nonlinear deterministic and stochastic systems with unbounded delay," Taiwanese Journal of Mathematics, vol. 14, no. 5, pp. 1777-1797, 2010. 


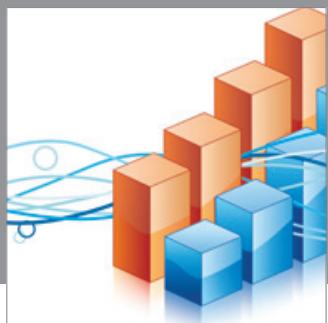

Advances in

Operations Research

mansans

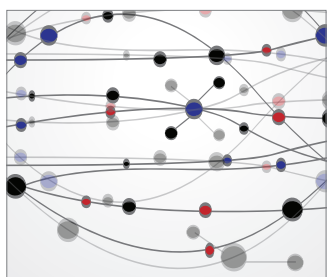

The Scientific World Journal
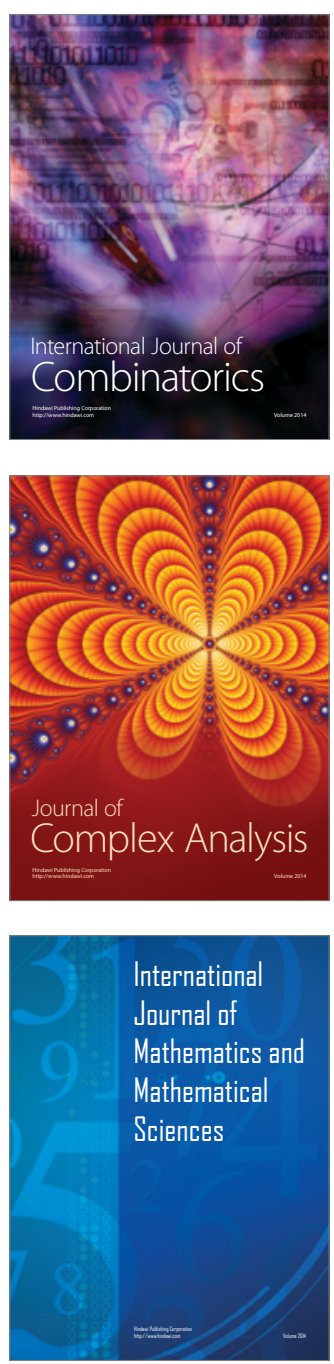
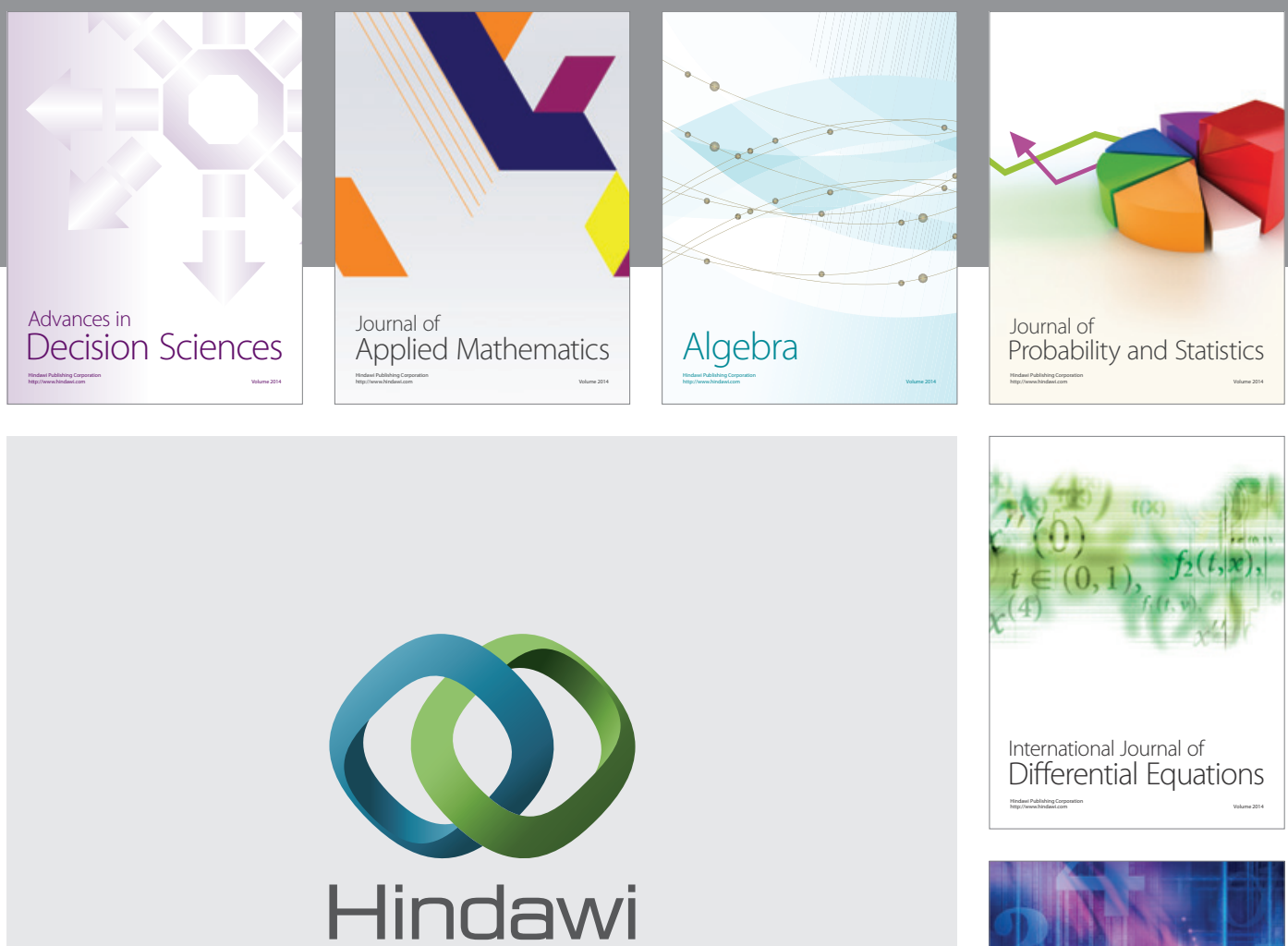

Submit your manuscripts at http://www.hindawi.com
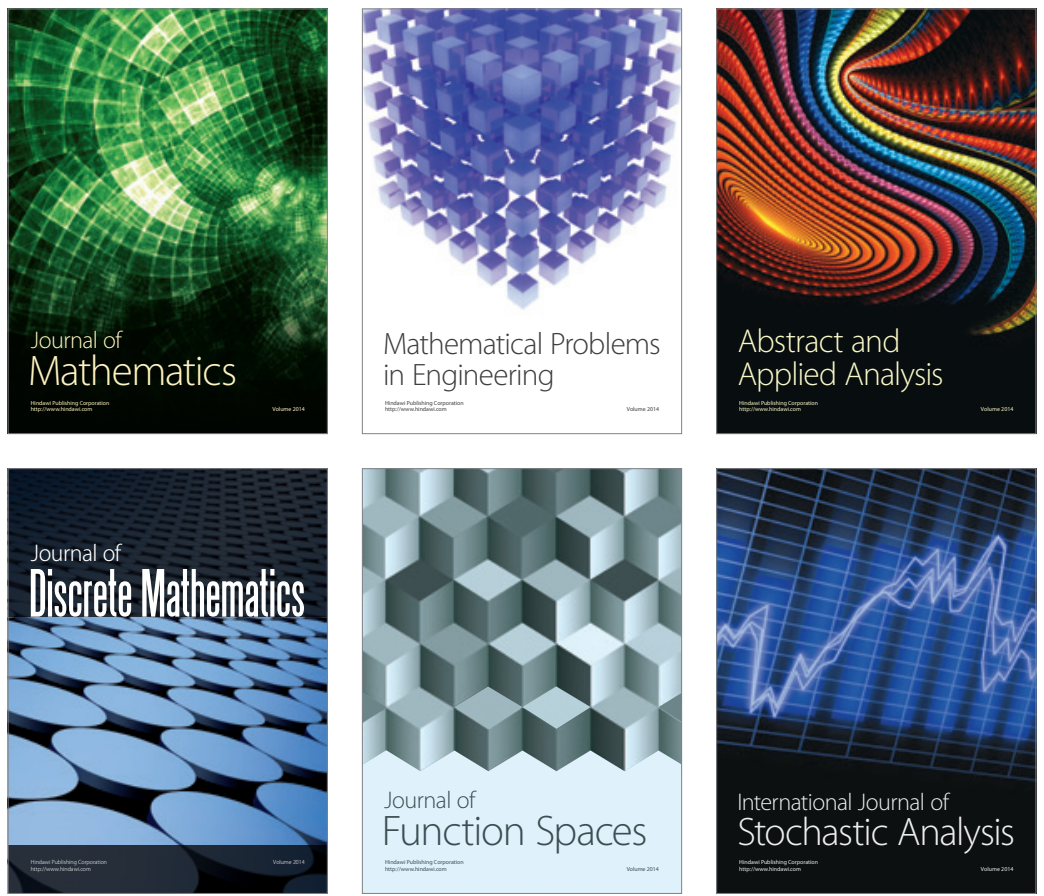

Journal of

Function Spaces

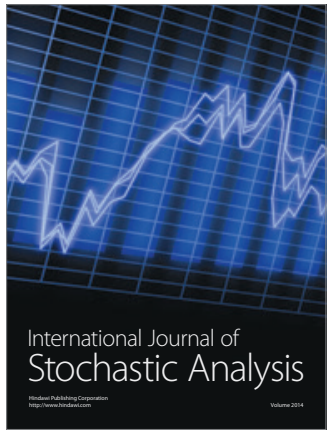

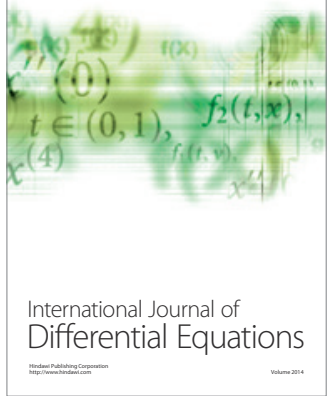
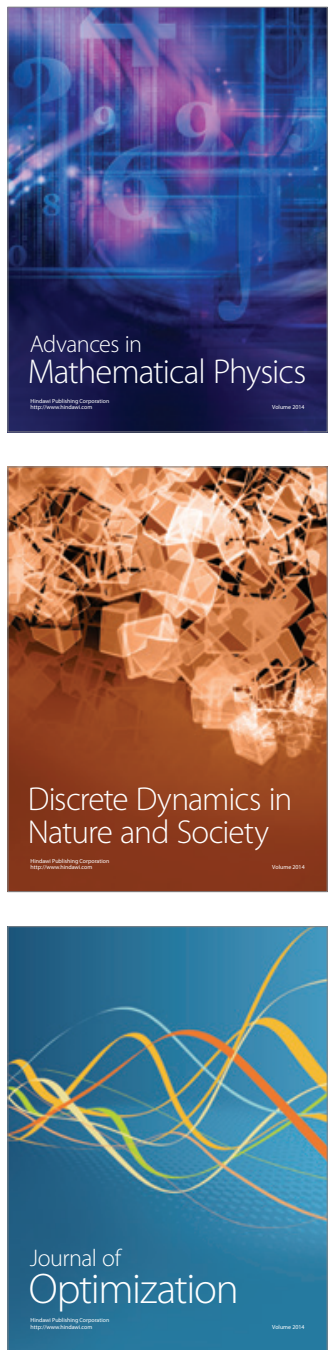